\title{
Electrical and Mechanical Properties of Graphite Nanosheet/Carbon Nanotubes-filled Epoxy Nanocomposites
}

\author{
Ki-Seok Kim ${ }^{1}$, Kyeong-Eun Choi $^{2}$ and Soo-Jin Park ${ }^{1, \uparrow}$ \\ ${ }^{1}$ Department of Chemistry, Inha University, Incheon 402-751, Korea \\ ${ }^{2}$ Dept. of Practical Arts Education, Jeonju National University of Education, Wansan-gu, Jeonju 560-757, Korea \\ ^e-mail: sjpark@inha.ac.kr \\ (Received November 22, 2009; Accepted December 13, 2009)
}

\begin{abstract}
In this work, the effect of co-carbon fillers on the electrical and mechanical properties of epoxy nanocomposites was investigated. The graphite nanosheets (GNs) and multi-walled carbon nanotubes (MWNTs) were used as co-carbon fillers. The results showed that the electrical conductivity of the epoxy nanocomposites showed a considerable increase upon an addition of MWNTs when GNs were fixed at $2 \mathrm{wt} . \%$. This indicated that low content GNs formed the bulk conductive network and then MWNTs added were intercalated between the GN layers, resulted in the formation of additional conductive pathway. Furthermore, the flexural strength of the epoxy nanocomposites was enhanced with increasing the MWNT content. It was probably attributed to the flexible MWNTs compared with rigid GNs, resulted in the enhancement of the mechanical properties.
\end{abstract}

Keywords : Epoxy, Graphite nanosheets, MWNTs, Nanocomposites, Electrical conductivity

\section{Introduction}

Recently, polymer nanocomposites reinforced with conductive fillers have attracted much attention due to their various application areas, such as electronic devices, polymer electrolyte membranes, anti-static devices, and gas sensors [1,2]. As fillers for conductive composites, carbonbased fillers, i.e., carbon black, graphite or carbon nanotubes are one type of commonly used materials capable of improving the physical, thermal, and electrical properties of polymers [3,4].

Among the carbon fillers, the natural graphites are composed of hexagonal carbon layers having a thickness in the range of 30 80 nm with excellent electrical conductivity of about $10^{4} \mathrm{~S} / \mathrm{cm}$. Therefore, graphites have received a considerable amount of attention in the graphites/polymer nanocomposites [5,6]. Also, expanded graphites (EGs) having a high aspect ratio can easily be obtained through an acid treatment and rapid heat treatment of natural graphites; polymer nanocomposites containing a low quantity of EGs have excellent electrical conductivity $[7,8]$.

Despite their many advantages, EGs are difficult to use in the preparation of polymer nanocomposites in a direct intercalation method, and nanocomposites based on EGs have a high degree of porosity because many micropores in EGs interrupt the filling polymer. Therefore, the nanoscale dispersion of EGs in a polymer matrix is very important to achieve EG/polymer nanocomposites $[9,10]$.
Recently, graphite nanosheets (GNs) created by powdering EGs have garnered much attention as nanoscale conductive fillers because they has a higher aspect ratio compared to that of EGs and tend to disperse easily in a polymer matrix. Polymer nanocomposites based on GNs have been reported; however, the inherently limited conductivity and rigidity of GNs restricts higher electrical and mechanical properties of conductive polymer composites [11,12].

Therefore, in the present study, GNs and MWNTs are used as the co-carbon fillers and epoxy nanocomposites are prepared using a melt mixing method. The effect of cocarbon fillers on the electrical and physical properties of epoxy nanocomposites is discussed.

\section{Experimental}

\subsection{Materials}

The natural graphites were supplied from Aldrich. Multiwalled carbon nanotubes (MWNTs) produced by chemical vapor deposition (CVD) process were obtained from Nanosolution Co. (Korea). The properties of MWNTs were: purity $>95 \mathrm{wt} \%$ and average diameter $10 \sim 25 \mathrm{~nm}$. The epoxy resins (YD-128) were supplied from Kukdo Chemical Co. (Korea). The epoxide equivalent weight and a density of DGEBA were $185 \sim 190 \mathrm{~g} / \mathrm{eq}$ and $1.16 \mathrm{~g} / \mathrm{cm}^{3}$ at $25^{\circ} \mathrm{C}$. The curing agent, 4,4'-diaminodiphenyl methane (DDM), was 
obtained from TCI. The 3-glycidoxypropyltrimethosysilane (GPTS) was supplied from Shin Etsu.

\subsection{Preparation of GNs and MWNTs}

EGs were produced by chemical oxidation using a sulfuric and nitric acid solution $(4: 1, \mathrm{v} / \mathrm{v})$ and a rapid heat-treatment at $1000^{\circ} \mathrm{C}$ for $90 \mathrm{~s}$. GNs were easily obtained by the powdering via ultrasonic treatment of EGs in acetone for $6 \mathrm{~h}$.

MWNTs were acid-treated using a 3:1 mixture of sulfuric acid and nitric acid at $60^{\circ} \mathrm{C}$ for $12 \mathrm{~h}$. After the filtering of the sample, MWNTs were washed with water until their $\mathrm{pH}$ was 7.0, and the washed samples were dried at $100^{\circ} \mathrm{C}$.

\subsection{Preparation of the nanocomposites}

The epoxy nanocomposites were prepared by a melt mixing method. The fixed $1 \mathrm{wt} \%$ GPTS and $2 \mathrm{wt} . \%$ GNs were dispersed in epoxy resins for $1 \mathrm{~h}$ with sonication and were then mixed for $3 \mathrm{~h}$ while stirring at $80^{\circ} \mathrm{C}$. After then, MWNTs were added from 0.25 to $1.0 \mathrm{wt} . \%$ in the GN/epoxy mixture with sonication for $1 \mathrm{~h}$ and melt-mixing at $80^{\circ} \mathrm{C}$ for $3 \mathrm{~h}$. The stoichiometric amount of curing agent was added to the MWNTs/GNs/epoxy mixtures. The mixtures were poured into a mold and cured at $120^{\circ} \mathrm{C}$ for $1 \mathrm{~h}$ and at $150^{\circ} \mathrm{C}$ for $2 \mathrm{~h}$. Finally, they were then postcured at 180 for $1 \mathrm{~h}$ in a convection oven.

\subsection{Characterization and measurements}

The morphologies of the EGs, GNs, MWNTs, and nanocomposites were observed via scanning electron microscopy (SEM, JEOL S-4200) and transmission electron microscopy (TEM, JEOL FE-TEM 2006).

Electrical conductivity of the nanocomposites was measured at room temperature using a four probe digital multimeter (MCP-T610, Mitsubishi Chem.).

The flexural properties (for the specimen size of $2 \mathrm{~mm} \times$ $25 \mathrm{~mm} \times 50 \mathrm{~mm}$ ) were measured by universal test machine (UTM, LLOYD, LR5K) according to ASTM D-790 and the span-to-depth ratio was 16:1.

\section{Results and Discussion}

The morphologies of the EGs, GNs, and MWNTs are shown in Fig. 1. Rapid heating of acid-treated graphites at a sufficiently high temperature caused an expansion by the escape of $\mathrm{H}_{2} \mathrm{SO}_{4}$ intercalated into the graphite interlayer. The EGs show a worm-like appearance, with a large interlayer distance by expansion. They have a loose and porous vermicular structure (Fig. 1 (a)). The EGs were converted to GNs (Fig. 1 (b)) by ultrasonication in a solvent, and the GNs
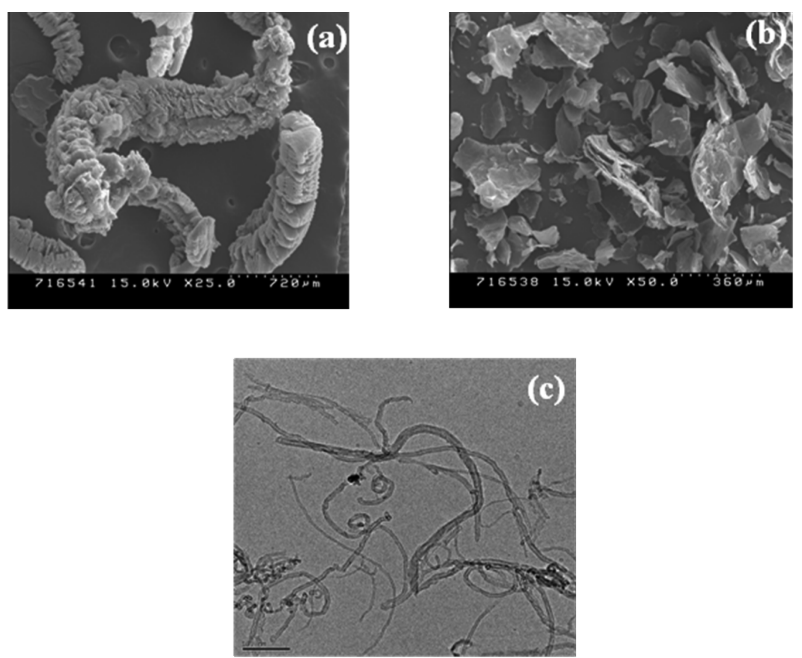

Fig. 1. Morphologies of the EGs (a), GNs (b), and MWNTs (c).

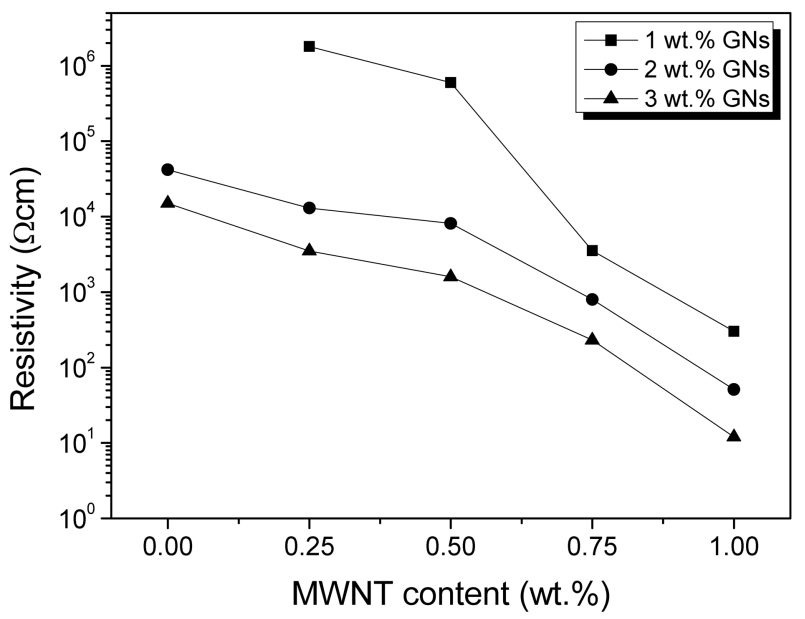

Fig. 2. Electrical conductivity of epoxy nanocomposites as a function of various GNs/MWNT ratios.

are composed of the thinner sheets having both one layer and multiple layers. The disentangled MWNTs after the acid treatment are shown in Fig. 1 (c).

The electrical conductivity is more important to qualify the nanocomposites for application as electrodes. This is influenced by the filler content, filler type, and dispersion of the filler in the polymer matrix. In this work, MWNTs with a low content of GNs are used as a combination to obtain high electrical conductivity of the nanocomposites through a synergic effect between carbon materials.

The electric conductivity of the nanocomposites at room temperature is showed in Fig. 2. The electric conductivity of the GN/epoxy nanocomposites is not measured up to $1 \mathrm{wt} . \%$ GNs (over the limit of equipments), and the percolation threshold is found to be 2 wt.\% GNs, resulting from the formation of a conductive network above $2 \mathrm{wt} . \%$ GNs. After fixing the $2 \mathrm{wt} . \% \mathrm{GNs}$, the electrical conductivity of the 

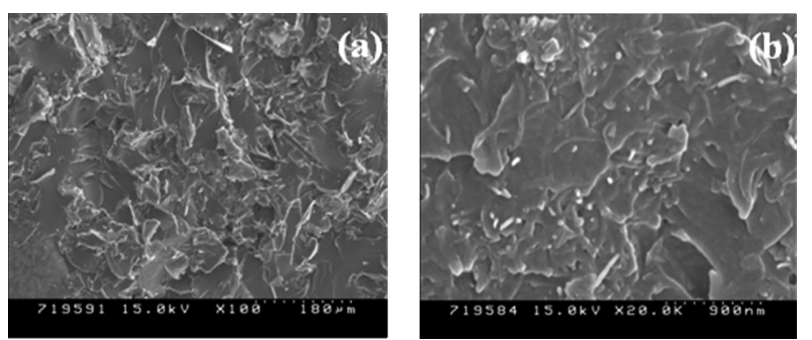

Fig. 3. SEM images of (a) GNs/epoxy and (b) 1 wt.\% MWNTs/ GNs/epoxy nanocomposites (2 wt.\% GNs was fixed).

epoxy nanocomposites increases with an addition of MWNTs, showing a remarkable increase at a content up to 1 wt.\% MWNTs. It is shown that GNs form the bulk conductive network and then highly intercalated MWNTs between the GN layers offer additional conductive pathway, leading to the combination effect of co-carbon materials.

In a previously reported study, the low content of 2Dgraphite nnoplates provide relatively high enhancement of the electrical conductivity compared with 1D-carbon nanotubes due to the higher dimension and high aspect ratio. However, the hybrid carbon fillers do not provide high synergistic effect to the electrical conductivity, which is attributed to the presence of thin polymer layer between GNs and MWNTs [13]. As shown in Fig. 2, at low content of $1 \mathrm{wt} . \%$ GNs, the epoxy nanocomposites show remarkably increased electrical conductivity with the addition of MWNTs due to high electrical conductivity of the MWNTs, whereas the effect of the addition of MWNTs at over 2 wt.\% GNs is lower compared with the epoxy nanocomposites containing $1 \mathrm{wt} . \%$ GNs $[14,15]$.

Fig. 3 shows the cross-section of the GNs/epoxy and 1 wt.\% MWNTs/GNs/epoxy nanocomposites. This SEM image indicates that GNs are shown to be homogeneously dispersed in epoxy resins. Also, MWNTs are uniformly dispersed in the GN/epoxy nanocomposites (Fig. 3 (b)) and interconnected between GN layers. GNs having large dimension form the bulk conductive network, resulting in the low electrical conductivity and then MWNTs which have large aspect ratio and electrical conductivity are intercalated into the GN layers. Low MWNT content can effectively offer the enhanced electrical conductivity of the nanocomposites, resulting from the densely conductive network by MWNTs intercalated in bulk GN network. Additionally, the combinational use of GNs and MWNTs reduces the total filler loading, which provides the high electrical properties of the nanocomposites [16,17].

The mechanical properties of the epoxy nanocomposites are measured to determine the effect of MWNT and GNs on the flexural strength $\left(\sigma_{\mathrm{f}}\right)$ and elastic modulus in flexure $\left(E_{b}\right)$. The flexural strength values of the nanocomposites are determined with the three-point bending test and are calculated as follows [18]:

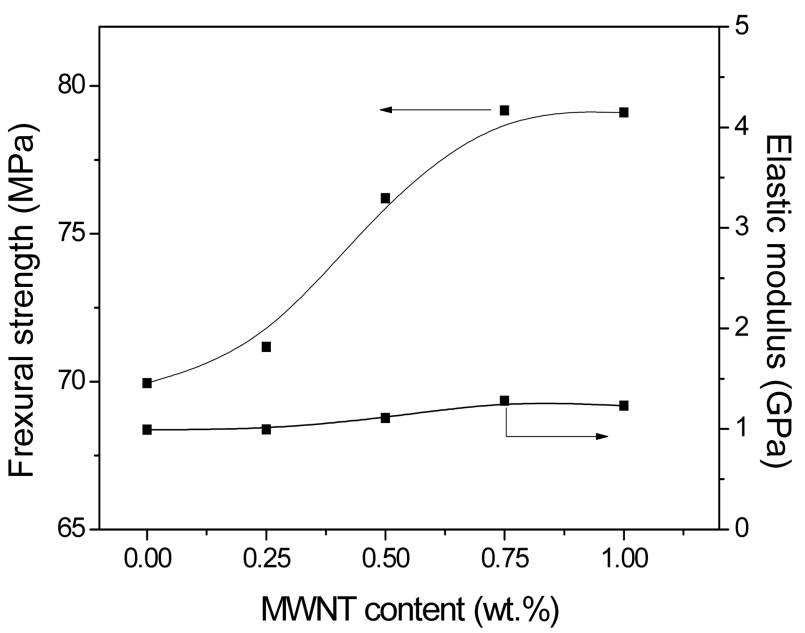

Fig. 4. Flexural strength and elastic modulus of the epoxy nanocomposites as a function of the MWNT content ( $2 \mathrm{wt} \% \mathrm{GNs}$ was fixed).

$$
\begin{aligned}
\sigma_{f} & =\frac{3 P L}{2 b d^{2}} \\
E_{b} & =\frac{L^{3}}{3 b d^{3}} \frac{\Delta P}{\Delta m}
\end{aligned}
$$

where $P$ is the applied load, $L$ the span length, $b$ the width of specimen, $d$ the thickness of the specimen, $\Delta P$ the change in force in the linear portion of the load-deflection curve, and $\Delta m$ is the change in deflection corresponding to $\Delta P$.

Fig. 4 shows the result of flexural strength $\left(\sigma_{f}\right)$ and elastic modulus $\left(E_{b}\right)$ values of the nanocomposites. As a result, the $\dot{o}_{\mathrm{f}}$ and $E_{b}$ values of the nanocomposites are increased with increasing the MWNT content up to $0.75 \mathrm{wt} . \%$, whereas GN/epoxy nanocomposites are brittle. This result means that the addition of flexible MWNTs compared with rigid GNs into the epoxy resins increases the ductility of the nanocomposites. However, the mechanical properties of the nanocomposites are slightly decreased above $1 \mathrm{wt} . \%$ MWNTs. It is probably attributed to the accumulation of MWNTs, resulting in weak interaction between the MWNTs and epoxy resins [19].

\section{Conclusion}

In this study, the effect of GNs and MWNTs on the electrical and mechanical properties of the epoxy nanocomposites was investigated. The co-carbon fillers dispersed in the epoxy resins were resulted in the formation of conductive network. The electrical conductivity of the nanocomposites was remarkably improved with increasing the MWNT content. This implied that the interconnected MWNTs between the GN layers provided an additional 
conductive pathway. Also, the flexural strength of the nanocomposites was increased with increasing the MWNT content, resulted from the addition of flexible MWNTs compared with rigid GNs.

\section{References}

[1] Zheng, W.; Wong, S. C.; Sue, H. J. Polymer 2002, 43, 6767.

[2] Zhao, Y. F.; Xiao, M.; Wang, S. J.; Ge, X. C.; Meng, Y. Z. Comp. Sci. Tech. 2007, 67, 2528.

[3] Celzard, A.; McRae, E.; Mareche, J. F.; Furdin, G.; Sundqvist, B. J. Appl. Phys. 1998, 83, 1410.

[4] Weng, W.; Chen, G.; Wu, D. Polymer 2005, 46, 6250.

[5] Celzard, A.; March, J. F.; Furdin, G.; Puricelli, S. J. Phys. D:Appl. Phys. 2000, 33, 3094.

[6] Park, S. J. "Interfacial Forces and Fields: Theory and Applications", ed. By J. P. Hsu, Marcel Dekker, New York, 1999, chapter 9.

[7] Chen, G. H.; Weng, W.G.; Wu, D. J.; Wu, C. L. Eur. Polym. J. 2003, 39, 2329.

[8] Uhl Fawn, M.; Yau, Q.; Nakajima, H.; Manias, E.; Wilkie, C. A. Polym. Degrad. Stabil. 2005, 89, 70.
[9] Du, X. S.; Xiao, M.; Meng, Y. Z.; Hay, A. S. Polymer 2004, $45,6713$.

[10] Chen, G.; Lu, J.; Wu, D. Mater. Chem. Phys. 2007, 104, 240.

[11] Kalaitzidou, K.; Fukushima, H.; Drzal, L. T. Comp. Sci. Tech. 2007, 67, 2045.

[12] Lu, W.; Lin, H.; Wu, D.; Chen, G. Polymer 2006, 47, 4440.

[13] Yu, A. Y.; Ramesh, P.; Sun, X.; Bekyarova, E.; Itkis, E.; Haddon, R. C. Adv. Mater. 2008, 20, 4740.

[14] Yu, A.; Ramesh, P.; Itkis, M. E.; Bekyarova, E.; Haddon, R. C. J. Phys. Chem. C 2007, 111, 7565.

[15] Shenogin, S.; Xue, L. P.; Ozisik, R.; Keblinski, P., Cahill, D. G. J. Appl. Phys. 2004, 95, 8136.

[16] Martin, C. A.; Sandler, J. K. W.; Shaffer, M. S. P.; Schwarz, M. K.; Bauhofer, W.; Schulte, K.; Windle, A. H. Comp. Sci. Tech. 2004, 64, 2309.

[17] Li, J.; Wong, P. S.; Kim, J. K. Mater. Sci. Eng. A 2008, $483,660$.

[18] Heo, S. I.; Oh, K. S.; Yun, J. C.; Jung, S. H.; Yang, Y. C.; Han, K. S. J. Power Sources 2007, 171, 396.

[19] Seo, M. K.; Lee, J. R.; Park, S. J. Mater. Sci. Eng. A 2005, 404,79 . 